\title{
Erratum to: Characterization of Nanoporous Surfaces as Templates for Drug Delivery Devices
}

\author{
Ashish Rastogi, ${ }^{1}$ Tanushree Bose, ${ }^{1}$ Marc D. Feldman, ${ }^{2,3}$ Devang Patel, ${ }^{2}$ and Salomon Stavchansky ${ }^{1,4}$
}

Published online: 17 November 2009

The following two errors were caught by the authors after publication of the article.

Table I.: At the end of the table title " $(\mathrm{N}=5)$ " should be " $(\mathrm{N}=3)$ ".

\section{Results and Discussion}

\section{Dimensional Analysis of Nanopores}

At the end of the fourth sentence (Table I represents the cumulative statistical data of the five nanoporous wafers.) in this section "five nanoporous wafers" should be "three nanoporous wafers".

\footnotetext{
${ }^{1}$ Division of Pharmaceutics, College of Pharmacy, The University of Texas at Austin, Austin, TX 78712, USA.

${ }^{2}$ Division of Cardiology, The University of Texas Health Science Center at San Antonio, San Antonio, TX 78229, USA.

${ }^{3}$ South Texas Veterans Affairs Health Care System, San Antonio, TX 78229, USA.

${ }^{4}$ To whom correspondence should be addressed. (e-mail: stavchansky@ mail.utexas.edu)
} 